\title{
Management of hyperglycaemia in type 2 diabetes: the 2018 consensus report by ADA/EASD
}

\section{Insights from one of the authors}

\author{
MELANIE J DAVIES 1,2
}

Key words: type 2 diabetes, hyperglycaemia, consensus report, adult

\section{Background to the consensus report}

On 5 October 2018 a consensus report by the American Diabetes Association (ADA)/European Association for the Study of Diabetes (EASD) on the management of hyperglycaemia in type 2 diabetes was presented at EASD in Berlin as well as being simultaneously published online in Diabetes Care and Diabetologia. ${ }^{1,2}$ The report was commissioned by the ADA and EASD to update the previous position statement that was published in 2015. ${ }^{3}$ Co-chaired by myself on behalf of the European team and by John Buse for the ADA, a writing group of 10 individuals representing both Europe and North America with a balanced gender mix and spectrum of declarations of interest with three members having no conflicts of interest was convened.

The writing group accepted the 2015 edition of the position statement as a starting point. ${ }^{3}$ However, we recognised with the rapidly evolving evidence base, particularly with regard to cardiovascular outcome trials (CVOTs), that a more structured review of the literature would be necessary and therefore a search was conducted on PubMed between 1 January 2014 and 28 February 2018 which included randomised trials, systematic reviews, metaanalysis on the effectiveness of safety pharmacological or nonpharmacological interventions in the management of people with type 2 diabetes. The search strategy is available via a link published in the publication. , $^{2}$

Different from the previous process was that the draft of the consensus was presented at the ADA in June 2018, reviewers were nominated by members of the writing group but also by the ADA's Professional Practice Committee and Executive Board as well as the

Diabetes Research Centre, University of Leicester, UK

Leicester Diabetes Centre, University Hospitals of Leicester NHS Trust, UK

Address for correspondence: Professor Melanie J Davies Diabetes Research Centre, University of Leicester, Leicester Diabetes Centre, Leicester General Hospital, Leicester LE5 4PW, UK

Tel: +44 (0)1162586481

E-mail: melanie.davies@uhl-tr.nhs.uk

Br J Diabetes 2018;18:137-140

https://doi.org/10.15277/bjd.2018.193
EASD's Committee of Clinical Affairs and approximately 35 invited reviewers reviewed the document and are acknowledged in the final manuscript. Furthermore, we received over 800 comments including those received after the ADA presentation of the draft consensus.

Looking back as to how these reports have developed over the last 12 years, in the 2006 original version only metformin, insulin, sulfonylureas and glitazones were available. ${ }^{4}$ In 2012 the algorithm developed but still focused mainly on drug therapy, but now included dipeptidyl peptidase-4 inhibitor (DPP-4i) and glucagon-like peptide-1 receptor agonists (GLP-1 RA) as therapeutic options. ${ }^{5}$ In 2015 the sodium-glucose cotransporter 2 inhibitors (SGLT-2i) were introduced and there was more of a focus on individualised therapy. ${ }^{3}$ Between 2015 and 2018 there has been a rapid evolution of the evidence base, particularly with regard to CVOTs, the results of over 15 now being in the public domain.

\section{Main changes}

The current consensus, following the comprehensive review of the literature from 2014, attempts to interpret this new evidence into a series of pragmatic and practical road maps to help the multidisciplinary team navigate the increasingly complex area of hyperglycaemic management in patients with type 2 diabetes. 1,2

Whilst our focus is on management of hyperglycaemia, holistic patient management with comprehensive cardiovascular risk management in its many forms is acknowledged as an essential and important part of diabetes management and all clinicians should be aware of the importance of multifactorial treatment in type 2 diabetes.

A key emphasis is the patient-centred approach to care, with the patient at the centre with the ultimate aim to both prevent complications and optimise their quality of life and creation of a 'decision cycle' to ensure patient-centred management (Figure 1). This begins by emphasising the importance of accessing key patient characteristics, highlighting specific factors which can impact on the choice of treatment, particularly the importance of considering adherence and persistence to therapy and reminding clinicians of the importance of a shared decision-making approach to create a management plan which should be agreed and then implemented.

Ongoing support and monitoring is not just around traditional activity such as self-monitoring of blood glucose and $\mathrm{HbA}_{1 c}$, but includes emotional wellbeing, and the decision cycle also highlights 
Figure 1. Decision cycle for patient-centred glycaemic management in type 2 diabetes

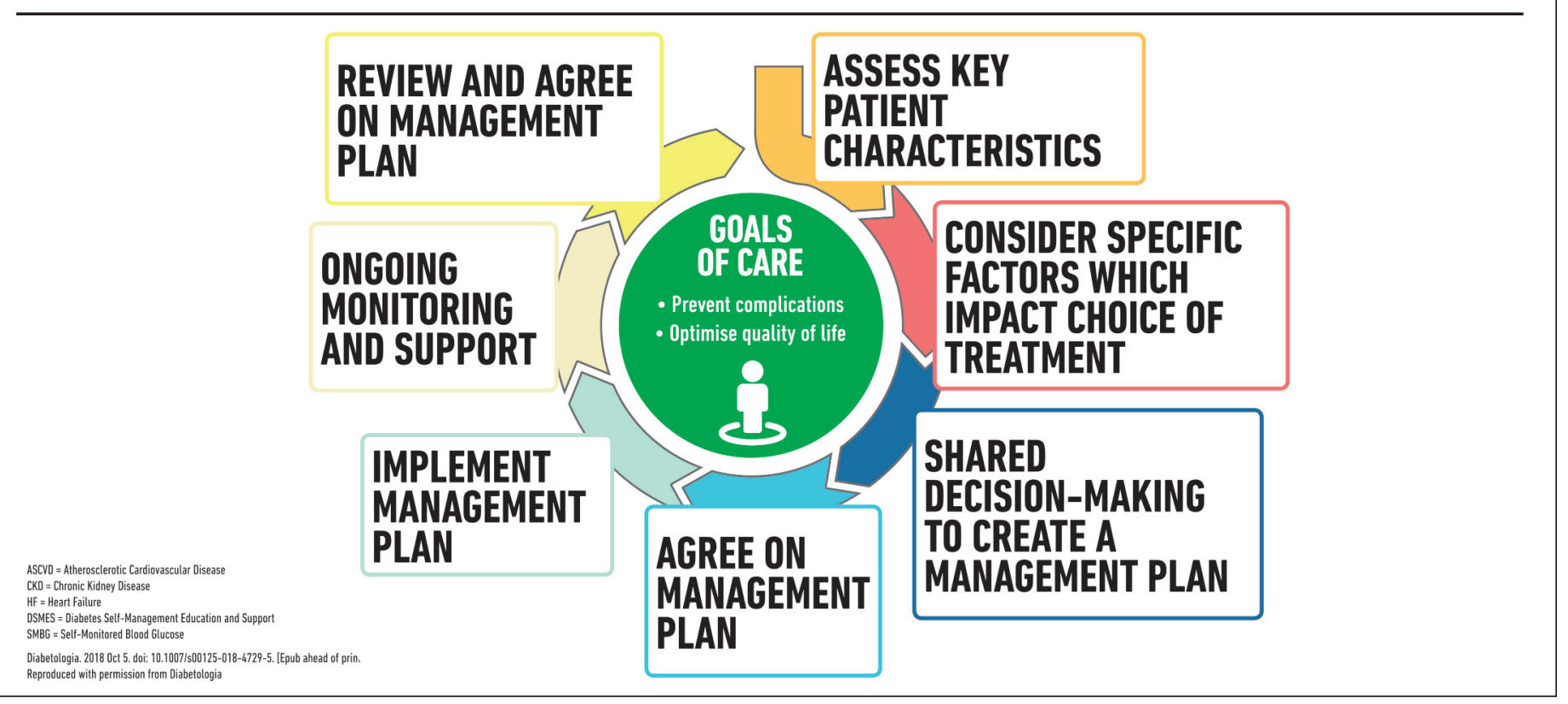

the importance of continuous review to avoid therapeutic inertia.

While there is obviously a focus on pharmacological interventions, there is a much greater focus on the importance of lifestyle interventions with a greater emphasis on weight loss, obesity management (including metabolic surgery) and effectiveness of physical activity interventions. A table in the report comprehensively reviews therapeutic options and includes lifestyle including diet quality, physical activity and energy restriction, highlighting the importance of these therapies in the continued management of people with type 2 diabetes. ${ }^{1,2}$

The preferred choices of glucose-lowering agents have been driven specifically by the new evidence from the CVOTs, but also we have taken a new approach of highlighting areas of particular need - for example, management of weight and the risk of hypoglycaemia - as well as being very cognisant of the increasing pressures that value for money and cost play in treatment choices.

The burden of evidence continues to suggest that foundational therapy is metformin as well as comprehensive lifestyle management. Regarding the role of Metformin as continued first line, this was based on long term knowledge of efficacy, safety, tolerability as considerations regarding low cost and data from the relatively small sub-study ( $\mathrm{n}=342$ treatment arm, 411 control arm) from UKPDS; though some have argued that it is a strength of that study that an effect was found from relatively small numbers. ${ }^{6}$ We did acknowledge that metformin's role as first-line medication may be a 'quirk of history' and with the rapid pace of new evidence may be challenged in future consensus reports. When choosing glucoselowering medication, we felt that an important early factor was the presence of established atherosclerotic cardiovascular disease (ASCVD) or chronic kidney disease (CKD). In those patients in whom ASCVD predominates, the suggestion of the addition of a GLP-1 RA or an SGLT-2i with proven cardiovascular benefit is preferred after metformin (Figure 2). We acknowledge that ASCVD is defined differently across the trials, but usually refers to those with established CVD - for example, myocardial infarction, stroke, revascularisation procedures or those at high cardiovascular risk. However, when heart failure (HF) or CKD predominates, we suggest the preferred use of an SGLT-2i with evidence of reducing HF and/or CKD progression if the estimated glomerular filtration rate (eGFR) is adequate. This recognises that patients with type 2 diabetes are at increased risk for HF and that there are significant and consistent reductions in hospitalisation for HF in the SGLT-2i trials. The impact on CKD progression is of particular interest, and the press release from the CREDENCE study was already in the public domain although results not fully disclosed. Whilst the impact on CKD progression was a secondary endpoint, the composite used is regarded as a 'gold standard' for CKD progression and was significantly and consistently reduced in both SGLT-2i studies EMPA-REG and CANVAS. The data on renal outcomes with the GLP-1 RAs liraglutide in the LEADER trial and semaglutide in the SUSTAIN trial were reassuring but were mostly driven by a reduction in albuminuria, so our preference in the consensus was for SGLT-2i over GLP-1 RA for reduction of CKD progression. One of the obvious gaps from evidence to practice is the evolving role of SGLT-2i to reduce CKD progression but the current licence indication for eGFR with SGLT-2i. The evidence is compelling for reducing CKD progression down to an eGFR of $30 \mathrm{~mL} / \mathrm{min} / 1.73 \mathrm{~m}^{2}$, however the licence indication often precludes their use. For example, in the UK, emagliflozin and canagliflozin can be used down to an eGFR of $45 \mathrm{~mL} / \mathrm{min} / 1.73 \mathrm{~m}^{2}$ and dapagliflozin only above $60 \mathrm{~mL} / \mathrm{min} / 1.73 \mathrm{~m}^{2}$. However, this is rapidly changing; for example, in Canada the licence for empagliflozin is already reduced down to $30 \mathrm{~mL} / \mathrm{min} / 1.73 \mathrm{~m}^{2}$ and is likely to change in other regions of the world.

It is important to recognise that the majority of the patients in the CVOTs were on a background of metformin, approximately 70-80\%, and that patients with established ASCVD, HF and CKD 
Figure 2. Choosing glucose-lowering medication in those with established atherosclerotic cardiovascular disease (ASCVD) or chronic kidney disease (CKD)

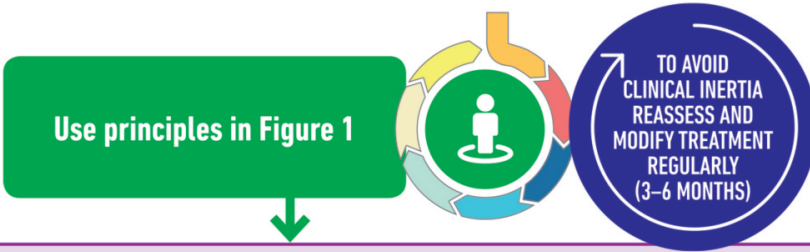

Use metformin unless contraindicated or not tolerated

If not at $\mathrm{HbA}_{1 \mathrm{c}}$ target:

- Continue metformin unless contraindicated (remember to adjust dose/stop metformin with declining eGFR)

- Add SGLT2i or GLP-1 RA with proven cardiovascular benefit ${ }^{1}$ (See below)

If at $\mathrm{HbA}_{\mathrm{1c}}$ target:

- If already on dual therapy, or multiple glucose-lowering therapies and not on an SGLT2i or GLP-1 RA, consider switching to one of these agents with proven cardiovascular benefit' (See below)

OR reconsider/lower individualised target and introduce SGLT2i or GLP-1 RA

OR reassess $H_{b A_{1 c}}$ at 3 month intervals and add SGLT2i or GLP-1 RA if $H_{b A}$ goes above target

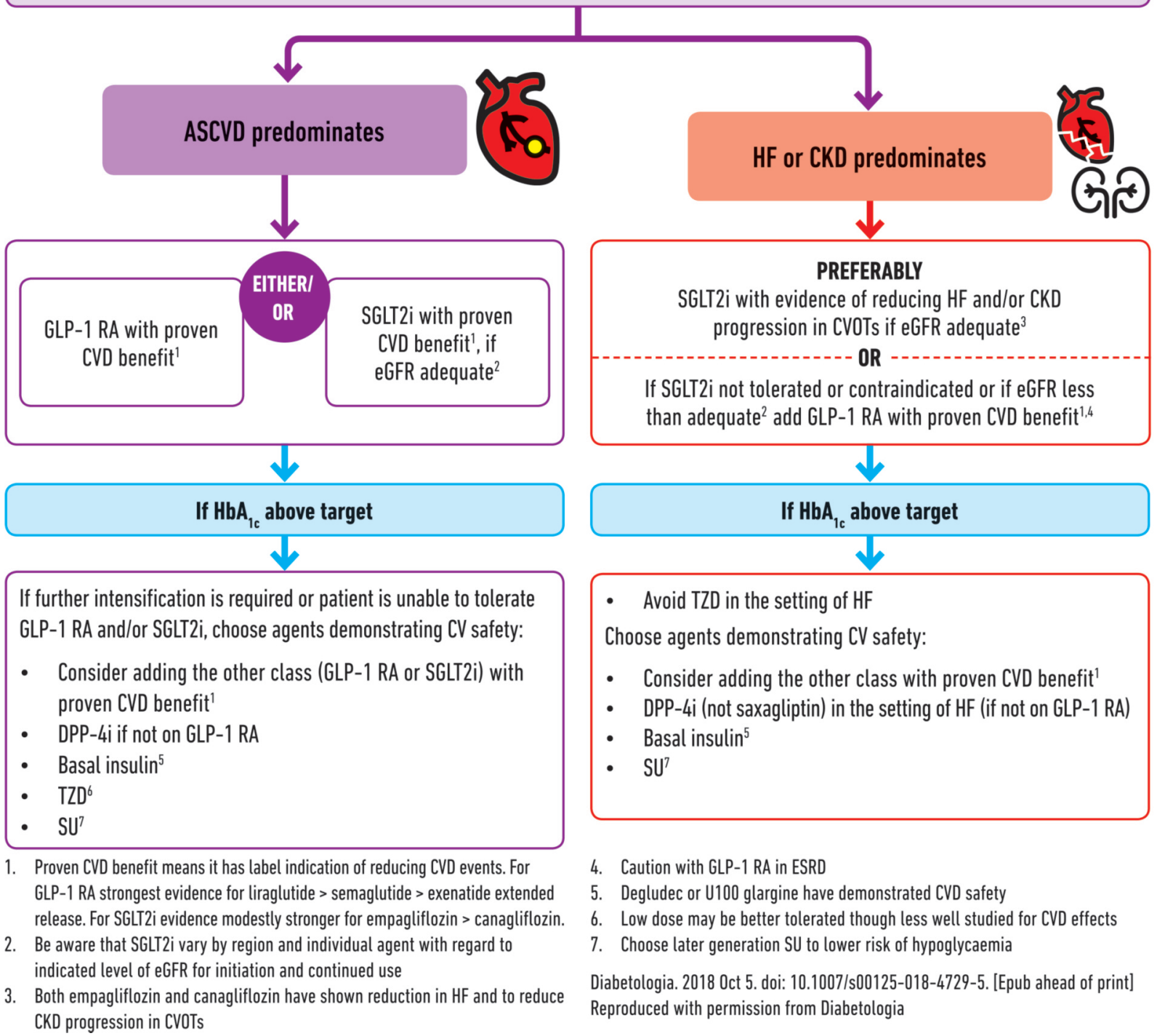


in aggregate probably account for approximately $25 \%$ of the type 2 diabetes population. Therefore, in the other $75 \%$, when it comes to consideration of glucose-lowering medication, we have 'called out' a compelling need to minimise hypoglycaemia and a compelling need to minimise weight gain or promote weight loss and a further consideration when cost is a major issue.

Another key change from the previous recommendation is the preferred use of GLP-1 RA in the majority of patients with type 2 diabetes who require the greater glucose-lowering effect of an injectable medication. The heterogeneity in the class of GLP-1 RA with regard to CVOT outcome - but in particular the magnitude of

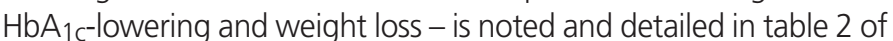
the consensus report. In the report we have given quite a detailed road map to injectable therapy with particular emphasis on the importance of initiation and titration and practical guidance as to how to intensify insulin, but also using combinations of insulin with GLP-1 RA. This was because of the evolving evidence in this area while also recognising therapeutic inertia, particularly with regard to insulin therapy in type 2 diabetes where many patients remain with poor glycaemic control on under-titrated therapy or conversely an increasing number of patients on complex insulin regimens where actually de-intensification of therapy may be appropriate.

\section{Future directions and potential gaps}

The document highlights some of our key knowledge gaps which will require increased research and investment. These include better segmentation of type 2 diabetes, better data on the optimum approaches to management in frail and older patients, which is urgently required particularly considering the controversy around glycaemic targets in the benefits and harms of a specific treatment, the rationale for early combination therapy, the new and emerging questions from the recent CVOT studies, particularly whether the benefits extend to those in the lower risk population, whether there is any added benefit of the combined use of GLP-1 and SGLT-2 inhibitors. All of this is framed in the issue around access and cost of therapies and in the context of a rapidly changing field in terms of the availability and use of technology and telemedicine.

\section{Relevance to the UK}

The consensus report is in marked contrast to the most recent NICE guideline which was published in December 2015 and then updated in 2017 with a further review in July 2017, which felt that there was insufficient evidence to include any data or recommendation regarding the CVOTs. 7,8 This contrasts markedly with other regions of the world. The ADA standard of care has adopted the consensus report, Diabetes Canada has also updated its guideline, and IDF and the European Society of Cardiology have all updated their guidance with regard to including data from CVOTs.

There is a risk that England in particular will be significantly behind our European and North American partners when it comes to guidance regarding the management of type 2 diabetes.

The risk of any consensus recommendations is that they quickly become out of date, and the press releases from both DECLARE and CREDENCE were considered within this report but there are other CVOTs in the pipeline including CAROLINA, which is the head to head of a DPP-4i with a sulfonylurea, and PIONEER 6, which is with an oral GLP-1 RA. The ADA will be adopting a 'live update' approach to review and incorporate any new significant evidence as it emerges.

There is also the importance of considering the management of the frail elderly person and highlighting the importance of de-intensification of therapy in the context of therapeutic inertia, which was considered in the consensus but should have been given more prominence. Nevertheless, we think the overall approach of this patient-centred decision-making to support consistent efforts to improve diet and exercise, but with the addition of glucoselowering medications based on patient co-morbidities and clinical concerns, is helpful in a world where management of hyperglycaemia in type 2 diabetes has become extraordinarily complex.

Conflict of interest Professor Melanie Davies has acted as consultant, advisory board member and speaker for Novo Nordisk, Sanofi-Aventis, Lilly, Merck Sharp \& Dohme, Boehringer Ingelheim, AstraZeneca and Janssen, an advisory board member for Servier and as a speaker for Mitsubishi Tanabe Pharma Corporation and Takeda Pharmaceuticals International.

She has received grants in support of investigator and investigator-initiated trials from Novo Nordisk, Sanofi-Aventis, Lilly, Boehringer Ingelheim and Janssen.

Funding Professor Melanie Davies received funding from EASD/ADA to support her travel expenses and accommodation for the writing group meetings in the UK and USA and attend the ADA/EASD meetings in 2018.

Disclaimer The author informs readers that the views, thoughts and opinions expressed in the text belong solely to the author and not necessarily to the author's employer, organisation, committee or other group or individual.

\section{References}

1. Davies MJ, D'Alessio DA, Fradkin J, et al. Management of hyperglycemia in type 2 diabetes, 2018. A consensus report by the American Diabetes Association (ADA) and the European Association for the Study of Diabetes (EASD). Diabetes Care 2018 Oct 4. https://doi.org/10.2337/dci18-0033 [Epub ahead of print]

2. Davies MJ, D'Alessio DA, Fradkin J, et al. Management of hyperglycaemia in type 2 diabetes, 2018. A consensus report by the American Diabetes Association (ADA) and the European Association for the Study of Diabetes (EASD). Diabetologia 2018 Oct 5 https://doi.org/10.1007/s00125-018-4729-5 [Epub ahead of print].

3. Inzucchi S, Bergenstal RM, Buse JB, et al. Management of hyperglycemia in type 2 diabetes, 2015: a patient-centered approach: update to a Position Statement of the American Diabetes Association and the European Association for the Study of Diabetes. Diabetes Care 2015;38:140-9. https://doi.org/10.2337/dc14-2441.

4. Nathan D, Buse JB, Davidson MB, et al. Management of hyperglycemia in type 2 diabetes: a consensus algorithm for the initiation and adjustment of therapy. A consensus statement from the American Diabetes Association and the European Association for the Study of Diabetes. Diabetes Care 2006:29:1963-72. https://doi.org/10.2337/dc06-9912

5. Inzucchi S, Bergenstal RM, Buse JB, et al. Management of hyperglycaemia in type 2 diabetes: a patient-centered approach. Position statement of the American Diabetes Association (ADA) and the European Association for the Study of Diabetes (EASD). Diabetologia 2012;55:1577-96. https://doi.org/10.1007/s00125-012-2534-0

6. https://www.bmj.com/content/352/bmj.h6748/rapid-responses

7. National Institute for Health and Care Excellence. Type 2 diabetes in adults: management. Guideline [28]. 2 December 2015, updated May 2017. https://www.nice.org.uk/guidance/ng28.

8. National Institute for Health and Care Excellence. Type 2 diabetes in adults: management. NICE guideline 'Evidence reviews for SGLT-2 inhibitors and GLP-1 mimetics'. March 2018. https://www. nice.org.uk/ guidance/ng28/evidence/march-2018-evidence-reviews-for-sglt2-inhibitors-and-glp1-mimetics-pdf-4783687597 